\title{
Secagem da Madeira de Louro Preto (Nectandra cuspidata) em Estufa de Micro-ondas
}

\author{
Anízio de Araújo Cavalcante ${ }^{1,2}$, Ricardo Manfredi Naveiro ${ }^{1,3}$, Suely Souza Costa ${ }^{4}$ \\ ${ }^{1}$ Escola Superior de Tecnologia - EST, Universidade do Estado do Amazonas - UEA, Manaus/AM, Brasil \\ ${ }^{2}$ Universidade do Estado do Amazonas - UEM, Manaus/AM, Brasil \\ ${ }^{3}$ Instituto Alberto Luiz Coimbra de Pós-graduação e Pesquisa de Engenharia - COPPE, Universidade Federal do Rio de \\ Janeiro - UFRJ, Rio de Janeiro/RJ, Brasil \\ ${ }^{4}$ Instituto Nacional de Pesquisas da Amazônia - INPA, Manaus/AM, Brasil
}

\begin{abstract}
RESUMO
A secagem por micro-ondas em um futuro próximo irá desempenhar importante papel na industrialização da madeira, pois dela depende a melhora da estabilidade dimensional e aumento da resistência mecânica, dentre outras. O objetivo deste trabalho foi avaliar a secagem da madeira de louro preto utilizando o processo por micro-ondas e sua influência na formação de rachaduras, no tempo de secagem e na umidade final. Foi utilizado o programa desenvolvido para a madeira de mogno em função da semelhança entre os valores de suas densidades básicas. Foram realizadas 3 secagens, tendo a secagem S1 com umidade inicial média acima do PSF após $2 \mathrm{~h} 25$ min chegado aos 13,99\% de umidade final média. As secagens S2 e S3 com umidade inicial média abaixo do PSF depois de 2 h 08 min e 2 h 02 min apresentaram 14\% e 14,62\% de umidade final média, respectivamente. As secagens foram consideradas rápidas, e a diferença de umidade inicial das amostras mostrou que sofreram influência dos tratamentos na formação dos defeitos de rachaduras de topo e de superfície. A secagem S2 mostrou-se mais propensa em apresentar rachaduras de superfície do que rachaduras de topo, enquanto as rachaduras internas não se formaram em nenhuma das secagens realizadas.
\end{abstract}

Palavras-chave: secagem por micro-ondas, qualidade, defeitos.

\section{Louro Preto (Nectandra cuspidata) Wood Drying in Microwave Oven}

\begin{abstract}
Microwave drying will play an important role in the industrialization of timber in the near future, because improved dimensional stability and increased mechanical strength of wood are dependent on its drying. The objective of this study was to evaluate the drying of Louro preto wood using the microwave process and its influence on drying time, final moisture content, and formation of checks. A program originally developed for Mahogany wood was used due to the similarity between the basic density values of the two wood species. To this end, three (3) drying processes were conducted: drying S1, with initial average moisture content above the fiber saturation point (FSP), reached $13.99 \%$ of the final average moisture content after $2 \mathrm{~h}$ 25min; whereas drying S2 and S3, with initial average moisture contents below the FSP, showed 14.00 and $14.62 \%$ of the final average moisture content after $2 \mathrm{~h} 8 \mathrm{~min}$ and $2 \mathrm{~h} 2 \mathrm{~min}$, respectively. The drying processes were considered fast, and the difference in the initial moisture contents of the samples showed the influence of the treatments in the formation of end and surface checks. Drying S2 was more prone to present surface checks than end checks, whereas internal checks were not found in any of the drying processes performed.
\end{abstract}

Keywords: microwave drying, quality, defects. 


\section{INTRODUÇÃO}

Um novo processo de secagem de madeiras com a utilização de micro-ondas está sendo desenvolvido pelo Instituto Nacional de Pesquisas da Amazônia no âmbito do Programa Madeiras da Amazônia - INPA/INCT. Esta tecnologia é recente no Brasil, mas na Europa os primeiros relatórios acerca de sua utilização foram publicados entre 1950 e 1960 (Leiker et al., 2011). Porém, o seu uso para fins industriais, em processos de cozimento e secagem, começou a partir dos anos de 1940, empregando as frequências de 2450 e $915 \mathrm{MHz}$ (Resch, 2006). De acordo com Lundgren (2005), o número de aplicações comerciais para a utilização de micro-ondas tem aumentado para uma variada gama de aplicações como aquecimento, comunicações, medições etc.

Uma característica da secagem por micro-ondas é o aquecimento de dentro para fora, gerando calor diretamente no centro da madeira. Por isso, o mecanismo da rotação dipolar é dominante para materiais com alta quantidade de água livre (Mcloughlin et al., 2003), pois quando o material dielétrico é exposto a micro-ondas, os íons, átomos e moléculas são excitados e movem-se sob a influência do campo elétrico, produzindo calor que é transferido neste processo. Em um material com alta quantidade de água livre, muitas moléculas são normalmente orientadas aleatoriamente (Gao, 2010), porém o campo elétrico tende a alinhar com a rotação campo - dipolo. Com as flutuações do campo, estes processos de alinhamento e relaxamento ocorrem milhões de vezes por segundo, convertendo a energia elétrica em energia potencial e em seguida em calor.

As potenciais vantagens da secagem por micro-ondas podem ser verificadas em três características (Mays, 2005). Em primeiro lugar, as micro-ondas penetram no interior dos materiais e depositam diretamente a energia, ou seja, o calor a ser gerado em todo o volume do material. É possível, neste caso, conseguir o aquecimento rápido de muitos materiais. Em segundo lugar, além do aquecimento volumétrico, a estufa de micro-ondas pode ser utilizada para aquecimento seletivo. Materiais muito úmidos normalmente têm um fator de perdas dielétricas relativamente alto em comparação com os outros componentes, por isso a secagem por micro-ondas pode ser intrinsecamente autorregulável, levando ao fenômeno de nivelamento automático da umidade e, finalmente, a secagem eficiente. Em terceiro lugar, a energia de micro-ondas usa potência apenas quando necessária e está disponível instantaneamente, tornando o processo mais adequado para o controle automático.

De maneira geral, é importante ressaltar que, em uma secagem por micro-ondas, o feixe de energia atua no núcleo do corpo a ser aquecido, apresentando um efeito que causa a pressão osmótica, ou seja, uma pressão interna como se fora um êmbolo empurrando a água do centro para a periferia, uma vez que a vibração das moléculas é maior no interior da madeira (Ghandehr, 2005).

$\mathrm{O}$ aquecimento das estufas por micro-ondas apresenta um menor efeito na profundidade de penetração, quando a madeira está na temperatura ambiente e principalmente quando a madeira está muito úmida, porém este penetra mais profundamente na madeira congelada (Hansson \& Antti, 2003). Quando o tamanho do material a ser aquecido é maior que a profundidade de penetração da radiação, é esperado que a distribuição de temperatura não seja uniforme (Resch, 2006).

$\mathrm{O}$ tratamento térmico utilizando micro-ondas é promissor, pois oferece oportunidade para o processamento de secagem da madeira em um curto espaço de tempo. Antti (1999) esclarece que a energia de micro-ondas pode ser utilizada com sucesso para o aquecimento rápido e secagem de madeira e de acordo com Torgovnikov \& Vinden (2009), aumenta sua permeabilidade. Além disso, Seyfarth et al. (2003) mostram que a aplicação de micro-ondas reduz o tempo de secagem.

Egner \& Jagfeld (1964) e Resch (1968) esclarecem que este processo foi bem investigado na década de 1960, porém nas décadas de 1980 e 1990 foi estudado com muito mais atenção.

No entanto, a necessidade de se obter melhor compreensão da secagem por micro-ondas vem provocando o progresso teórico e questões práticas, $\mathrm{o}$ que tem levado à constante melhoria dos equipamentos de secagem (Antti, 1999). Contudo, na indústria da madeira este método ainda não está desenvolvido, em função de uma série de dificuldades técnicas. Problemas de distribuição não homogênea de micro-ondas, além da falta de ferramentas capazes de realizar a medição da umidade da madeira no interior da estufa, são alguns 
dos impedimentos a serem resolvidos (Seyfarth et al., 2003).

A primeira estufa de micro-ondas foi desenvolvida para madeira menos densa que, mesmo após uma secagem primária, ainda se apresentava muita úmida (Resch, 2006). Para Egner \& Jagfeld (1964) e Resch (1968), a tecnologia de aquecimento e secagem por micro-ondas pode ser aplicada aos produtos florestais, principalmente para tábuas finas (Resch, 1968; Mcalister \& Resch, 1971). O emprego de micro-ondas na secagem proporciona benefícios como a rápida conversão de energia em calor, penetração instantânea no material, absorção seletiva de energia chegando, sobretudo a zonas de maior umidade, secagem mais uniforme, acelera a cinética de secagem e qualidade superior àquela obtida pelos métodos convencionais (Decareau \& Peterson, 1986). A ressecagem por micro-ondas previne o excesso da secagem e mantém a aparência de qualidade igual ou melhor do que a ressecagem com ar quente (Resch et al., 1970).

Observa-se que tanto a secagem convencional quanto a secagem por micro-ondas são dependentes de determinadas características da madeira buscando a boa qualidade do produto final. A madeira de louro preto apresenta densidade básica de $0,48 \mathrm{gcm}^{-3}$, retração tangencial de $9,9 \%$, radial de $3,8 \%$, porém um fator de anisotropia (T/R) de 2,61 (Jankowsky et al., 1990). Segundo Durlo \& Marchiori (1992), esse fator é o índice mais importante para se avaliar a estabilidade da madeira, porém Rocha (2000) esclarece que o fator tomado de forma isolada não caracteriza a estabilidade dimensional, pois é necessária a verificação das contrações tangencial e radial. Durlo \& Marchiori (1992) mostram que valores do fator de anisotropia acima de 2 são considerados ruins.

No que se refere ao estado de conhecimento da secagem por micro-ondas de madeiras no Brasil, a revisão da literatura permite concluir que, devido à deficiência de estudos, há um reduzido número de artigos publicados sobre o assunto, além de como esta tecnologia de secagem está sendo executada, indicando que mais pesquisas devem ser exploradas no sentido de melhor conhecimento e divulgação. Para cobrir este vazio, a presente pesquisa teve como objetivo avaliar o comportamento da madeira de louro preto em relação ao tempo de secagem, assim como os defeitos de rachaduras e umidade final.

\section{MATERIAL E MÉTODOS}

A madeira utilizada foi o louro preto (Lauraceae - Nectandra cuspidata, NEES; MART), identificada no Laboratório de Anatomia da Madeira do INPA/INCT - Madeiras da Amazônia. A madeira foi doada pela Indústria de Pisos da Amazônia - IPA, localizada no município de Iranduba, AM. Foram realizadas 3 secagens com 12 tábuas por experimento com as dimensões nominais de 2,5 × 25×95 cm em espessura, largura e comprimento, respectivamente.

Inicialmente, a secagem foi realizada por meio do controle visual da qualidade em todas as tábuas, a fim de verificar a existência de rachaduras de topo, de superfície e de qualquer tipo de empenamentos. Ressalta-se que há pouca informação sobre o comportamento da secagem, assim também como critério na obtenção de um conjunto de amostras para a experimentação. Deste modo, o critério para a utilização da madeira de louro preto foi baseado em seu emprego, pois a espécie pode ser empregada para móveis, folhas faqueadas decorativas, construção naval, pequenos brinquedos, entre outros (Remade, 2011).

A estufa de micro-ondas utilizada é do tipo Easydry, com capacidade para $0,7 \mathrm{~m}^{3}$ de madeira empilhada, fabricada com tecnologia alemã, pela Wu. M Gobler (Figura 1). Este tipo de estufa possui inserido um software de programas padrões de secagens para 17 espécies de origem europeia, sendo os programas passíveis de serem modificados.

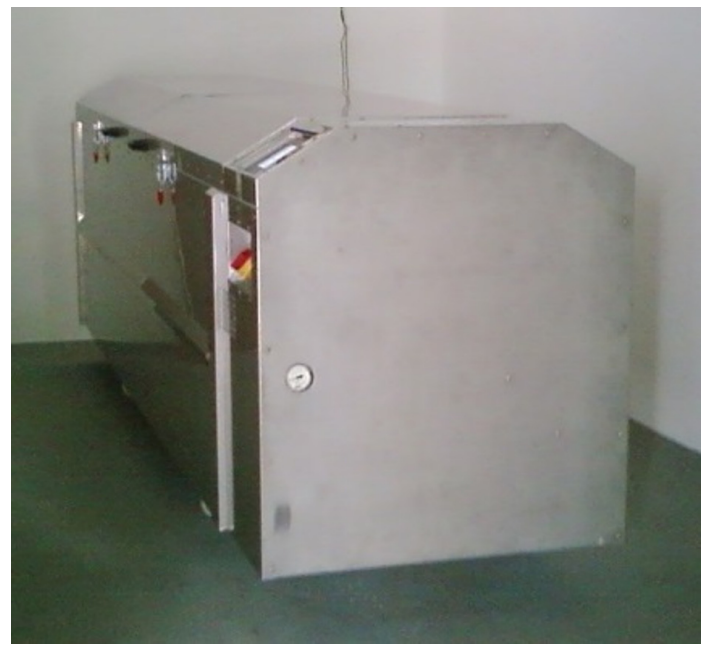

Figura 1. Estufa de micro-ondas utilizada no experimento. Figure 1. Microwave oven used in the experiment. 
A estufa de micro-ondas possui uma balança interna na qual o peso total da carga é determinado. Em função da umidade inicial medida com aparelho elétrico, da umidade final desejada e do peso determinado, o software instalado na estufa de micro-ondas calcula o tempo de aquecimento, tempo de secagem e tempo de resfriamento. Para comprovar se a umidade final da estufa estava de acordo com o proposto no programa de secagem, foi realizada a determinação dessa umidade a partir do método gravimétrico. Durante a pesquisa foram realizadas 3 secagens da madeira de louro preto com os seguintes teores de umidades inicial $(\mathrm{S} 1=48,87 \% ; \mathrm{S} 2=29,45 \%$ e S3 $=20,68 \%)$.

Em função da densidade básica do louro preto foi escolhido o programa para a espécie mogno (Tabela 1). Por um lado, observa-se, ainda, que a umidade de equilíbrio da região oscila em torno dos $17,1 \%$ (Galvão, 1975). Por outro lado, conforme Brotero (1941), a umidade da madeira utilizada em ambientes internos varia entre $10 \%$ e $13 \%$; neste estudo avaliou-se a umidade final de $10 \%$ para madeira seca.

As temperaturas, tanto inicial como final de secagem, foram programadas manualmente em $45^{\circ} \mathrm{C}$. Enquanto pelo software da estufa de micro-ondas foi determinado o tempo de aquecimento de $10 \mathrm{~min}$ e o tempo de resfriamento de $1 \mathrm{~h}$. Sendo todo o processo de secagem monitorado pelo painel de controle.

Após a secagem, na Tabela 2, as rachaduras foram classificadas e avaliadas de acordo com os critérios estabelecidos por Cavalcante (2013).
Neste estudo, a quantificação da incidência externa de rachaduras foi realizada na superfície e no topo, no final de todas as secagens (S1, S2, S3). Entretanto, as rachaduras internas, após avaliação final, não foram observadas.

O estudo foi conduzido em delineamento inteiramente ao acaso, com 12 repetições por secagens, com temperatura fixa de $45^{\circ} \mathrm{C}$. Os dados percentuais tanto de umidade inicial como de umidade final foram transformados em Arco seno $(\times / 100)$, a fim de torná-los mais homogêneos (Bartlett, 1936). Depois foram realizadas a análise de variância (ANOVA) e a análise dos modelos de regressão (pelo teste $\mathrm{F}$ ). A comparação de médias foi realizada pelo teste de Tukey. Em ambos os testes foi considerado o nível de $5 \%$ de significância. Depois de realizada a comparação do teste, a tabela de médias percentuais originais foi associada às comparações apresentadas com dados transformados. Todas as análises estatísticas foram realizadas por meio do software Minitab versão 14 (Minitab Inc, 2003).

\section{RESULTADOS E DISCUSSÃO}

Na Tabela 3 apresentam-se a umidade inicial (Ui), a umidade final (Uf) e o tempo de secagem (ts) para as condições testadas, onde foi observado que a primeira secagem durou 2 h 25 min, a segunda, 2 h 8 min e a terceira, 2 h 2 min. A umidade final da madeira programada em $10 \%$ alcançou níveis de 14\%, sendo, portanto, segundo Brotero (1941), umidade próxima

Tabela 1. Programa utilizado para a secagem da madeira de louro preto (Nectandra cuspidata).

Table 1. Schedule used in the Louro preto (Nectandra cuspidata) wood drying.

\begin{tabular}{cccccc} 
Aq (min) & $\operatorname{Ti}\left({ }^{\circ} \mathbf{C}\right)$ & $\operatorname{Tf}\left({ }^{\circ} \mathbf{C}\right)$ & Uri (\%) & Urf (\%) & Resf (h) \\
\hline 10 & 45 & 45 & 85 & 45 & 1 \\
\hline
\end{tabular}

Aq = Aquecimento; $\mathrm{Ti}$ = Temperatura inicial; $\mathrm{Tf}=$ Temperatura final; Uri = Umidade relativa inicial; Urf = Umidade relativa final; Resf $=$ Resfriamento .

Tabela 2. Critérios utilizados na classificação de tendência ao aparecimento de rachaduras.

Table 2. Criteria used in classifying trend of checks appearance.

\begin{tabular}{|ccc|}
\hline Percentual de peças com defeito (\%) & Classe & Tendência \\
\hline $0-15$ & 1 & Ausente \\
$16-31$ & 2 & Pequena \\
$32-47$ & 3 & Moderadamente pequena \\
$48-63$ & 4 & Média \\
\hline $64-79$ & 5 & Moderadamente grande \\
$80-100$ & 6 & Grande \\
\hline
\end{tabular}


daquela usada em ambientes internos, que varia entre $10 \%$ e $13 \%$.

Observa-se que as 3 secagens apresentaram diferentes umidades iniciais. A S1 apresentou maior umidade inicial $(48,87 \%)$ do que a S2 $(29,45 \%)$ e a S3 (20,68\%). Ressalta-se que o Programa de Secagem utilizado, ou seja, temperatura inicial e final de $45^{\circ} \mathrm{C}$, mostrou que os tempos necessários para alcançar a umidade final de aproximadamente $14 \%$ podem ser considerados próximos e também muito rápidos. Para a secagem S1, o tempo necessário de secagem foi superior aos demais assim como a umidade inicial, porém apresentou, juntamente com a $\mathrm{S} 2$, as menores umidades finais. Provavelmente, a secagem S1 pelo fato de ter apresentado alto teor de umidade mostrou maior fator de perda dielétrica, absorvendo mais energia e por isso provocando uma cinética de secagem mais rápida. Porém, Gao (2010) encontrou resultados diferentes, quando pesquisou a secagem de carvão vegetal em um processo convencional e por micro-ondas. As secagens S2 e S3 apresentaram menores tempos de secagem e

Tabela 3. Resultados da secagem por micro-ondas da madeira de louro preto (Nectandra cuspidata).

Table 3. Result of the microwave drying of Louro preto (Nectandra cuspidata) wood.

\begin{tabular}{cccc} 
Secagens & $\begin{array}{c}\text { Ui Média } \\
(\%)\end{array}$ & $\begin{array}{c}\text { Uf Média } \\
(\%)\end{array}$ & $\begin{array}{c}\text { ts } \\
\text { (h) }\end{array}$ \\
\hline S1 & 48,87 & 13,99 & $2 \mathrm{~h}^{\prime} 5^{\prime}$ \\
S2 & 29,45 & 14,00 & $2 \mathrm{~h}^{\prime}$ \\
S3 & 20,68 & 14,62 & $2 \mathrm{~h} 02^{\prime}$ \\
\hline
\end{tabular}

Ui = Umidade inicial; Uf = Umidade final; ts = tempo de secagem. menores diferença de umidade inicial e final, contudo a secagem S3 com menor umidade inicial e menor diferença de umidades, apresentou maior umidade final, contrapondo o resultado encontrado para a S1.

O comportamento dos tempos de secagem durante os tratamentos pode ter sido influenciado por variações encontradas na amostra, como por exemplo, quantidade de cerne da madeira, composição química, entre outros (Tsoumis, 1991). Os tempos de secagem obtidos nesta pesquisa estão em concordância com os estudos de Vongpradubchai \& Rattanadecho (2009) quando realizaram a comparação de micro-ondas com a secagem convencional. Os autores verificaram que o tempo foi de $12 \mathrm{~h}$ para menos de $1 \mathrm{~h}$, em madeiras de coníferas.

Vinden \& Torgovnikov (2000), estudando a secagem por micro-ondas de uma conífera, obtiveram uma redução no tempo de secagem na ordem de 2 a 10 vezes quando comparado à secagem convencional, e sem desenvolvimento de defeitos.

A Tabela 4 apresenta as avaliações das secagens da madeira louro preto e os valores dos critérios dos defeitos analisados em função do número de tábuas.

Anteriormente às secagens, procedeu-se o controle de qualidade e verificou-se que as tábuas estavam isentas de defeitos. Porém, após a secagem, a análise mostrou que não houve a presença de rachaduras internas, ocorrendo principalmente rachaduras de superfície e de topo. As rachaduras internas não surgiram devido à não formação do colapso ou do endurecimento superficial de forma severa (Cavalcante,

Tabela 4. Avaliação dos defeitos de secagem por micro-ondas para a madeira louro preto (Nectandra cuspidata). Table 4. Evaluation of microwave drying defects of Louro preto (Nectandra cuspidata) wood.

\begin{tabular}{|c|c|c|c|c|c|c|c|}
\hline \multirow{2}{*}{ Defeitos } & \multirow{2}{*}{$\begin{array}{c}\text { Experimentos } \\
\text { Classificação dos defeitos }\end{array}$} & \multicolumn{2}{|c|}{ S1 } & \multicolumn{2}{|c|}{ S2 } & \multicolumn{2}{|c|}{ S3 } \\
\hline & & $n^{\circ}$ & $\%$ & $\mathrm{n}^{\circ}$ & $\%$ & $\mathrm{n}^{\circ}$ & $\%$ \\
\hline Rachaduras internas & 1 & 12 & 100,0 & 12 & 100,0 & 12 & 100,0 \\
\hline$\%$ Total de defeito & & & $\mathbf{0 , 0}$ & & $\mathbf{0 , 0}$ & & $\mathbf{0 , 0}$ \\
\hline \multirow{5}{*}{ Rachaduras de superfície } & 1 & 6 & 50,0 & 5 & 41,7 & 6 & 50,0 \\
\hline & 2 & 3 & 25,0 & 3 & 25,0 & 3 & 25,0 \\
\hline & 3 & 2 & 16,7 & 1 & 8,3 & 2 & 16,7 \\
\hline & 4 & 1 & 8,3 & 1 & 8,3 & 1 & 8,3 \\
\hline & 5 & 0 & 0,0 & 2 & 16,7 & 0 & 0,0 \\
\hline$\%$ Total de defeito & & & 50,0 & & 58,3 & & 50,0 \\
\hline \multirow{2}{*}{ Rachaduras de topo } & 1 & 8 & 66,7 & 9 & 75,0 & 8 & 66,7 \\
\hline & 2 & 4 & 33,3 & 3 & 25,0 & 4 & 33,3 \\
\hline$\%$ Total de defeito & & & 33,3 & & 25,0 & & 33,3 \\
\hline
\end{tabular}

$\mathrm{n}^{\circ}=$ número de peças utilizadas; $\mathrm{S} 1$ = Secagem 1; S2 = Secagem 2; S3 = Secagem 3 . 
1991), indicando que, provavelmente, a temperatura utilizada na secagem pode ter sido considerada suave para o desenvolvimento de tal defeito.

Analisando rachaduras de superfície, verificou-se que as secagens $\mathrm{S} 1$ e $\mathrm{S} 3$ proporcionaram metade das peças ( 6 tábuas) sem defeito, enquanto a S2 apresentou 41,7\% (5 tábuas). Porém, ao nível de classificação de peças com defeito conforme descrito na metodologia observou-se que tanto a secagem S1 quanto a S3 apresentaram defeitos variando de pequeno a médio. A secagem S2 que apresentou 58,3\% (7 tábuas) com rachaduras de superfície mostrou-se mais propensa em função da classificação que variou de médio a moderadamente grande.

Dentre as secagens, a que apresentou maior quantidade de peças sem rachaduras de topo foi a S2 (75\%), seguida da S1 (66,7\%) e S3 (66,7\%). Considerando que a proporção de amostras com rachaduras de topo apresentou valores iguais e superiores a $25 \%$, mostram uma classificação considerada de pequena a moderadamente pequena.

Quando se relacionou a umidade inicial, verificou-se que as secagens $\mathrm{S} 1(48,87 \%)$ e S3 $(20,68 \%)$ apresentaram comportamento semelhante em relação aos defeitos de rachaduras de superfície e de topo, diferentemente da S2 (29,45\%). Apesar da diferença percentual da umidade entre as secagens, esse comportamento pode ter ocorrido em função da movimentação da madeira entre a direção radial e a direção tangencial a partir da secagem, pois são oriundas das características intrínsecas da espécie, tais como anatomia, gradiente de umidade, tensões de secagem, entre outros (Martins, 1988).

A secagem/tratamento $S 2$ que apresentou a umidade inicial próxima do PSF mostrou-se mais propensa à formação de rachaduras de superfície quando comparada às secagens $\mathrm{S} 1 \mathrm{e} \mathrm{S} 3$. Porém, ao se analisar a rachadura de topo verificou-se que esta mesma secagem/tratamento apresentou menor percentual. De acordo com Torgovnikov \& Vinden (2010), dependendo da espécie de madeira, da espessura e do teor de umidade inicial, o processo com energia por micro-ondas é recomendado, todavia existe a possibilidade do desenvolvimento de rachaduras internas e externas, afetando a propriedade de resistência da madeira e como consequência a sua desvalorização (Machado, 2006). Foi difícil tentar relacionar a tendência aos defeitos apresentados com o teor de umidade inicial, porém, segundo Leiker et al.
(2011), a eficiência de secagem em não apresentar defeitos parece depender principalmente dessa umidade. Segundo o mesmo autor, a secagem para amostras de faia com aproximadamente $50 \%$ de umidade inicial alcançou cerca de $70 \%$ de peças sem defeito, enquanto amostras com umidade inicial superior a $20 \%$ foram secas com uma eficiência de $80 \%$ de amostras sãs. Porém, em uma secagem convencional (a vapor), o louro preto apresentou comportamento moderado com poucos defeitos (Jankowsky et al., 1990).

Kubler (1987) esclarece que a temperatura aumenta na madeira úmida, comumente no intervalo de $110^{\circ} \mathrm{C}$ a $158^{\circ} \mathrm{C}$, amolecendo a lignina, hemicelulose e celulose, proporcionando o relaxamento do estresse. Torgovnikov \& Vinden (2010) mostram que a pressão do vapor gerado no interior da madeira rompe o tecido mais macio, criando micro e macro rachaduras, levando a uma melhor permeabilidade e consequente redução do estresse interno. É possível afirmar que o efeito temperatura não foi uma causa para a formação das rachaduras de superfície, pois a temperatura utilizada no experimento foi de $45^{\circ} \mathrm{C}$.

Experiências realizadas por Torgovnikov \& Vinden (2010) mostram que a secagem por micro-ondas numa frequência de $2,45 \mathrm{GHz}$ resultou em rachaduras de superfície menores quando comparadas com uma frequência de 0,922 GHz. Harris et al. (2008), ao analisarem algumas folhosas incluindo Eucalyptus pilularis, E. saligna, E. Regnans e E. marginata, notaram que a um baixo grau de intensidade de micro-ondas as madeiras com espessura de 30 a $50 \mathrm{~mm}$ não apresentaram diferença significativa na quantidade e tamanho de rachaduras de superfície entre as amostras secas em condições semelhantes de secagem. Com essas informações, pode-se inferir que, provavelmente, a madeira de louro preto tenha apresentado mediana tendência a rachaduras de superfície devido à sua espessura de $2,5 \mathrm{~cm}$, pois Resch (1968) esclarece que a secagem por micro-ondas é ainda mais bem aplicada à tábua fina, com espessura menor do que $2 \mathrm{~cm}$.

$\mathrm{Na}$ Tabela 5 encontram-se as médias do total de rachaduras de superfície e de topo dos três tratamentos.

Na comparação de média pelo teste de Tukey não foram detectadas diferenças estatísticas ao nível de significância de 5\%. Essa secagem, se executada dentro de técnicas adequadas, pode ocasionar pouca ou nenhuma degradação na madeira, apesar de a 
Tabela 5. Análise estatística das rachaduras de superfície e de topo.

Table 5. Statistical Analysis of end / surface checks.

\begin{tabular}{|c|c|c|c|}
\hline \multicolumn{2}{|c|}{ Rachaduras de superfície } & \multicolumn{2}{|c|}{ Rachaduras de topo } \\
\hline Secagem & $\begin{array}{c}\text { Ocorrência } \\
\%\end{array}$ & Secagem & $\begin{array}{c}\text { Ocorrência } \\
\%\end{array}$ \\
\hline S2 & $58,3 a$ & S1 & $33,3 a$ \\
\hline S1 & $50,0 \mathrm{a}$ & S3 & $33,3 a$ \\
\hline S3 & $50,0 \mathrm{a}$ & S2 & $25,0 \mathrm{a}$ \\
\hline
\end{tabular}

Médias seguidas pela mesma letra não diferem entre si, pelo teste de Tukey a $5 \%$ de probabilidade.

madeira de louro preto apresentar média tendência à formação de rachaduras de superfície, e com relação à rachadura de topo mostrou pequena predisposição. A secagem S2 com 58,3\% de peças com rachaduras de superfície apresentou uma diferença matemática quando comparada às S1 (50\%) e S3 (50\%). Contudo, na comparação de média pelo teste de Tukey, as secagens tiveram o mesmo comportamento semelhante, ou seja, mostraram que a ocorrência das rachaduras de superfície apresentadas pela espécie de louro preto mostrou-se propensa ao defeito. Por outro lado, ao se analisarem as rachaduras de topo, notou-se que as secagens $\mathrm{S} 1$ e $\mathrm{S} 3$, ambas com 33,3\% do referido defeito, apresentaram uma ocorrência maior do que a S2, com 25\%. Comportamento igual à análise anterior foi encontrado também para o exame das rachaduras de topo, significando que pela comparação de médias tiveram desempenho semelhante, porém a tendência que esta espécie apresentou na ocorrência da rachadura de topo foi considerada menor que a rachadura de superfície.

Em geral, as secagens efetuadas não mostraram comportamento distinto, em função das umidades iniciais ( $\mathrm{S} 1=48,87 \%$; $\mathrm{S} 2=29,45 \%$; $\mathrm{S} 3=20,68 \%$ ), pois todas apresentaram os mesmos índices de rachaduras de superfície e de topo. É importante notar que, apesar de a madeira de louro preto ter um fator de anisotropia de 2,61 (Jankowsky et al., 1990), as secagens apresentaram média tendência em relação às rachaduras de superfície e de pequena em relação à de topo. De acordo com Durlo \& Marchiori (1992), as variações dimensionais e a anisotropia são características indesejáveis da madeira, limitando o seu uso para diversas finalidades, e, segundo Masserann \& Mariaux (1985), a contração e a distinta expansão nas diferentes direções de crescimento são atribuídas, principalmente, à estrutura da parede celular e, em parte, à presença dos raios que dificultam a variação no sentido radial. Verifica-se ainda que o método por micro-ondas pode ser mais bem estudado, visando soluções que comportem diminuir os defeitos durante a secagem do louro preto e outras madeiras também.

\section{CONCLUSÕES}

O presente trabalho permite concluir que:

- a secagem do louro preto por micro-ondas pode ser considerada rápida;

- as umidades finais alcançadas estão dentro dos limites de uso interno e abaixo da umidade de equilíbrio (18\%) da cidade de Manaus;

- a madeira de louro preto, independente da umidade inicial, apresentou média propensão em apresentar rachaduras de superfície e pequena tendência em apresentar rachaduras de topo;

- as rachaduras internas não se formaram em função da temperatura de secagem não ter sido excessivamente elevada;

- em geral, os diferentes teores de umidade inicial e a temperatura de secagem influenciaram na formação das rachaduras de superfície e de topo.

\section{STATUS DA SUBMISSÃO}

Recebido: 21 ago., 2012

Aceito: 22 mar., 2016

\section{AUTOR(ES) PARA CORRESPONDÊNCIA}

\section{Anízio de Araújo Cavalcante}

Coordenação de Engenharia Florestal, Escola Superior de Tecnologia - EST, Universidade do Estado do Amazonas - UEA, Avenida Darcy Vargas, 1200, Parque Dez, CEP 69050-020, Manaus, AM, Brasil

e-mail: aacavalcante@uea.edu.br

\section{REFERÊNCIAS}

Antti L. Heating and drying wood using microwave power [tese]. Skelleftea: Luleå University of Technology; 1999.

Bartlett MS. The square root transformation in analysis of variance. Journal of the Royal Statistical Society 1936; 1(3): 68-78.

Brotero FA. Secagem da madeira em estufa. São Paulo: Instituto de Pesquisas Tecnológicas; 1941. 
Cavalcante AA. Ocorrência do colapso na secagem de madeiras de Eucalyptus grandis e Eucalyptus saligna [dissertação]. Piracicaba: Escola Superior de Agricultura Luiz de Queiroz, Universidade de São Paulo; 1991.

Cavalcante AA. Uma proposta de metodologia para secagem por micro-ondas de espécies madeireiras amazônicas [tese]. Rio de Janeiro: Instituto Alberto Luiz Coimbra, Universidade Federal do Rio de Janeiro; 2013.

Decareau RV, Peterson RA. Microwave processing and engineering. Chichester: Ellis Horwood; 1986.

Durlo MA, Marchiori JNC. Tecnologia da madeira: retratibilidade. Santa Maria: CEPEF/FATEC; 1992. Série Técnica n. 10.

Egner K, Jagfeld P. Versuche zur künstlichen Trocknung von Holtz durch Mikrowellen. Holtz-Zentralblatt 1964; 129: 297-300.

Galvão APM. Estimativas da umidade de equilíbrio da madeira em diferentes cidades do Brasil. IPEF 1975; (11): 53-65.

Gao F. Comparison of microwave drying and conventional drying of coal [dissertação]. Ontario: Queen's University Kingston; 2010.

Ghandehr. Ghandehr tecnologia avançada: o uso de micro-ondas e do Wawdry System na indústria madeireira [online]. Curitiba: Ghandehr; 2005 [citado em 2009 set 01]. Disponível em: http://www.ghandehr.com.br

Hansson L, Antti AL. Design and performance of an industrial microwave drier for on-line drying of wood components. In: Proceedings of the 8th International IUFRO Wood Drying Conference; 2003; Brasov, Romania. Brasov: IUFRO; 2003. p. 156-158.

Harris GG, Torgovnikov G, Vinden P, Brodie GI, Shaginov A. Microwave pretreatment of back sawn messmate boards to improve drying quality. Part 1. Drying Technol. 2008; 26(5): 579-584. http://dx.doi.org/10.1080/07373930801944770.

Jankowsky IP, Chimelo JP, Cavalcante AA, Galina ICM, Nagamura JCS. Madeiras brasileiras. Caxias do Sul: Spectrum; 1990.

Kubler H. Growth stress in trees and related wood properties. Forestry Abstr 1987; 48(3): 131-189.

Leiker M, Aurich K, Adamska MA. Accelerated drying of single hardwood boards by combined vacuum-microwave application. Nanjing: IUFRO; 2011. [citado 2011 jan 20]. Avaiable from: http://tudresden.de/die_tu_dresden/ fakultaeten/akultaet_maschinenwesen/ifvu/tvu/dteien/ pdfs/Accelerated_Drying.pdf.

Lundgren N. Modelling microwave measurements in wood [tese]. Skellefteå: Luleå University of Technology; 2005.

Machado RS. Effect of microwave treatment on oak compression strength. Silva Lusitana 2006; 14(1): 51-58.

Martins VA. Secagem de madeira serrada. Brasília: IBDF; $1988.56 \mathrm{p}$.
Masserann C, Mariaux A. Anisotropie de retrait et struture du bois - recherche d'influence des caractéres morphologiques transverses des fibres. Bois et Forêts des Tropiques 1985; 1985: 35-47.

Mays SLA. A practical comparison of moisture measuring systems. Hannover: Ligna; 2005.

Mcalister WR, Resch H. Drying 1-inch ponderosa pine lumber with a combination of microwave power and hot air. Forest Products Journal 1971; 21(3): 26-34.

Mcloughlin CM, Mcminn WAM, Magee TRA. Microwave drying of multi-component powder systems. Drying Technology 2003; 21(2): 293-309. http://dx.doi.org/10.1081/ DRT-120017750.

Minitab Inc. Meet Minitab para Windows ${ }^{\circledast}$ versão 14. State College: Minitab Inc; 2003.

Remade. 2011 [citado em 2011 dez 12]. Disponível em: http://www.remade.com.br/br/madeira_especies.php? num $=201 \&$ title $=$ Madeiras

Resch H. Microwaves for the drying of wood products. Holz als Roh- und Werkstoff 1968; 26(9): 317-324. http:// dx.doi.org/10.1007/BF02612806.

Resch H. High-frequency electric current for drying of wood: historical perspectives. Maderas: Ciencia y tecnologia 2006; 8(2): 67-82.

Resch H, Lofdahl CA, Smith FJ, Erb C. Moisture leveling in veneer by microwaves and hot air. Forest Products Journal 1970; 20(10): 50-58.

Rocha MP. Eucalyptus grandis Hill ex Maiden e Eucalyptus dunnii Maiden como fontes de matéria prima para serrarias [tese]. Curitiba: Universidade Federal do Paraná; 2000.

Seyfarth HR, Leiker M, Mollekopt M. Continuous drying of lumber in a vacuum kiln. In: Proceedings of the 8th International IUFRO; 2003; Brasov, Romania. Brasov: IUFRO; 2003. p. 159-163.

Torgovnikov G, Vinden P. High-intensity microwave wood modification for increasing permeability. Forest Products Journal 2009; 59(4): 84-92.

Torgovnikov G, Vinden P. Microwave wood modification technology and its applications. Forest Products Journal 2010; 60(2): 84-92. http://dx.doi.org/10.13073/00157473-60.2.173.

Tsoumis G. Science and technology of wood. New York: Chapman \& Hall; 1991

Vinden P, Torgovnikov G. The physical manipulation of wood properties using microwave. In: Proceedings of the International Conference of IUFRO. The Future of Eucalypts for Wood Production, 2000, Tasmania, Australia. Launceston: IUFRO; 2000. p. 240-247.

Vongpradubchai S, Rattanadecho P. The microwave processing of wood using a continuous microwave belt drier. Chemical Engineering and Processing: Process Intensification 2009; 48(5): 997-1003. http://dx.doi. org/10.1016/j.cep.2009.01.008. 